\title{
Application of a paste-type acellular dermal matrix for coverage of chronic ulcerative wounds
}

\author{
Minseok Jeon, So Young Kim \\ Department of Plastic and Reconstructive Surgery, Sanggye Paik Hospital, Inje University School of Medicine, Seoul, Korea
}

Background Chronic wounds occur due to failure of the normal healing process, associated with a lack of deposition of cellular components and a suitable microenvironment such as the extracellular matrix (ECM). Acellular dermal matrix (ADM) is viewed as an ECM substitute, and a paste-type ADM has recently been introduced. We hypothesized that CGPaste, an injectable paste-type ADM, could serve as a scaffold and promote wound healing.

Methods We retrospectively studied seven patients in whom CGPaste was applied between 2017 and 2018, who had pressure ulcers, necrotizing fasciitis, diabetic foot ulcers, traumatic defects, and osteomyelitis. The goal of applying CGPaste was to achieve complete wound healing with re-epithelialization or growth of granulation tissue, depending upon the wound bed status. CGPaste was injected based on the wound size along with the application of a dressing.

Results Four of the seven patients showed granulation tissue on their wound bed, while the other three patients had a bony wound bed. The mean wound area was $453.57 \mathrm{~mm}^{2}$ and the depth was $10.71 \mathrm{~mm}$. Wound healing occurred in five of the seven patients $(71.43 \%)$. The mean duration of complete healing was 2.4 weeks. Two patients showed failure due to paste absorption (29.57\%); these patients had wound beds comprising bone with relatively large and deep wounds ( $40 \times 30$ and $30 \times 20 \mathrm{~mm}^{2}$ in area and 15 and $10 \mathrm{~mm}$ in depth).

Conclusions CGPaste is an effective option for coverage of small and deep chronic wounds for which a flap operation or skin grafting is unfeasible.

Keywords Acellular dermis / Ulcer / Wound healing

\author{
Correspondence: So Young Kim \\ Department of Plastic and \\ Reconstructive Surgery, Sanggye Paik \\ Hospital, Inje University School of \\ Medicine, 1342 Dongil-ro, Nowon-gu, \\ Seoul 01757, Korea \\ Tel: +82-2-950-1114 \\ Fax: +82-2-932-6373 \\ E-mail:pajamakim@naver.com
}

Received: 31 May 2018 - Revised: 13 Sep 2018 - Accepted: 2 Oct 2018

pISSN: 2234-6163 • elSSN: 2234-6171 • https://doi.org/10.5999/aps.2018.00605 • Arch Plast Surg 2018;45:564-571

\section{INTRODUCTION}

A wound is generally considered to be chronic when it does not heal within 3 months [1]. Chronic wounds occur due to failure of the normal wound healing process, which is characterized by a well-coordinated, progressive series of interactions between cellular components and the microenvironment, including the extracellular matrix (ECM). Inflammatory and proteolytic activity damages the ECM and affects regenerative processes, with consequent destruction of mechanical skin integrity and the barrier function of skin [2]. Chronic wounds are known to occur in patients with underlying diseases such as diabetes mellitus (DM), peripheral arterial obstructive disease (PAOD), venous ulcers, or underlying osteomyelitis, all of which provide an environment potentially conducive to the interruption of normal wound healing. Such wounds present as narrow and deeply undermined defects in the form of sores, and it is challenging to promote wound healing and cover these chronic wounds be- 
cause they lack sufficient ECM and vascularity. The lack of ECM in chronic wounds presents a serious difficulty in wound healing because the ECM provides structural support and generates signals to modulate the compounds and processes that contribute to effective normal wound healing. Based on this concept, several attempts have been made to exchange the damaged tissue and to restore the function of the ECM in patients presenting with such wounds [2].

Conventional flaps could be an option to cover such chronic wounds; however, their use is limited because patients with chronic ulcerative wounds usually present with underlying diseases such as DM or PAOD that are associated with poor vascular status, and therefore have unacceptable recipient vessels. Furthermore, such patients are not suited to undergo prolonged surgery under general anesthesia because of their poor general condition. Additionally, coverage using a local flap is also associated with limitations in that the tissue adjacent to chronic wounds primarily comprises fibrotic scar tissue, and tissue from such donor sites is unsuitable for harvesting. Although skin grafting could be an alternative in such cases, effective engraftment may be unlikely in skin grafts placed over deep-seated wounds. Based on the aforementioned concept of replacement of the damaged ECM, the application of acellular dermal matrix $(\mathrm{ADM})$ is emerging as a useful substitute for the ECM in chronic wounds [2-4]. ADM is a biomaterial derived from human or bovine tissues that undergo processing and removal of cells with the retention of some portion of the ECM. ADM contains a framework of insoluble molecules such as collagen, elastin, and fibronectin. Such matrices retain signals that promote attachment, proliferation, and migration of surrounding host tissue $[2]$.

A paste-type ADM (CGPaste; CGBio, Inc., Seongnam, Korea) has been recently introduced. We hypothesized that the paste-type $\mathrm{ADM}$ could play an important role in providing an ECM scaffold and promoting cell integration and vascularization from the surrounding host tissue [5]. Through this mechanism, we expected that it could play an important role in re-epithelialization or promotion of granulation tissue growth. We investigated the clinical application and utility of paste-type $\mathrm{ADM}$ in patients presenting with chronic ulcerative wounds.

\section{METHODS}

\section{Material characteristics of paste-type ADM}

The paste-type ADM used in the present study was CGPaste (CGBio, Inc.), which is manufactured by crushing existing $\mathrm{ADM}$ material. It is an allograft derived from donated human skin. After removal of the subcutaneous fat layer, donated skin is

\section{Fig. 1. CGPaste in the syringe before use}

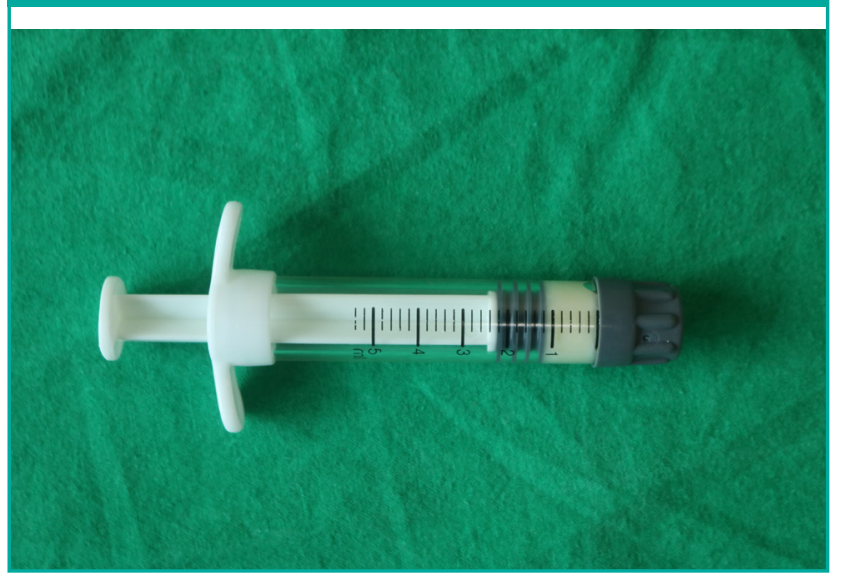

processed using a sodium chloride solution and detergent to remove the epidermis and all viable dermal cells while maintaining the original ECM. After the chemical treatment is completed, the ADM is crushed and processed into CGPaste. CGPaste is then packed in a syringe in $1 \mathrm{~mL}$ increments (from 1 to $5 \mathrm{~mL}$ ) that can be easily injected into ulcerative wounds (Fig. 1). It is recommended that the product be stored at room temperature, and its expiration date is a year from the date of manufacture.

\section{Methods}

We retrospectively analyzed seven patients with chronic ulcerative wounds who received treatment using CGPaste at the Department of Plastic and Reconstructive Surgery of Sanggye Paik Hospital between July 2017 and March 2018. This study was approved by the Institutional Review Board of Sanggye Paik Hospital (approval number: 2018-08-028). CGPaste was used in all patients after they provided informed consent regarding the cost and possibility of failure. The primary goal of applying CGPaste was to achieve adequate coverage of the exposed underlying wound bed, and the secondary goal was to ensure adequate re-epithelialization of open wounds. After confirming adequate surgical debridement and meticulous hemostasis, the CGPaste was injected into the ulcerative wound (Fig. 2). After application of the CGPaste to the chronic wound, negativepressure wound therapy (NPWT) was applied to the wound, and a wet gauze dressing was applied when NPWT was not available. In patients who received NPWT, the sponge was replaced every 3 to 4 days. A simple wet dressing was replaced every day or every other day to promote wound healing and the growth of granulation tissue. Six patients were inpatients and remained hospitalized until complete healing of the wound was confirmed.

The study outcomes assessed were achievement of complete wound healing, duration of wound healing, and the occurrence 


\section{Fig. 2. Application of CGPaste}

(A) Ulcerative wound on the dorsum of the foot before paste application. (B) Application of CGPaste to an ulcerative wound.
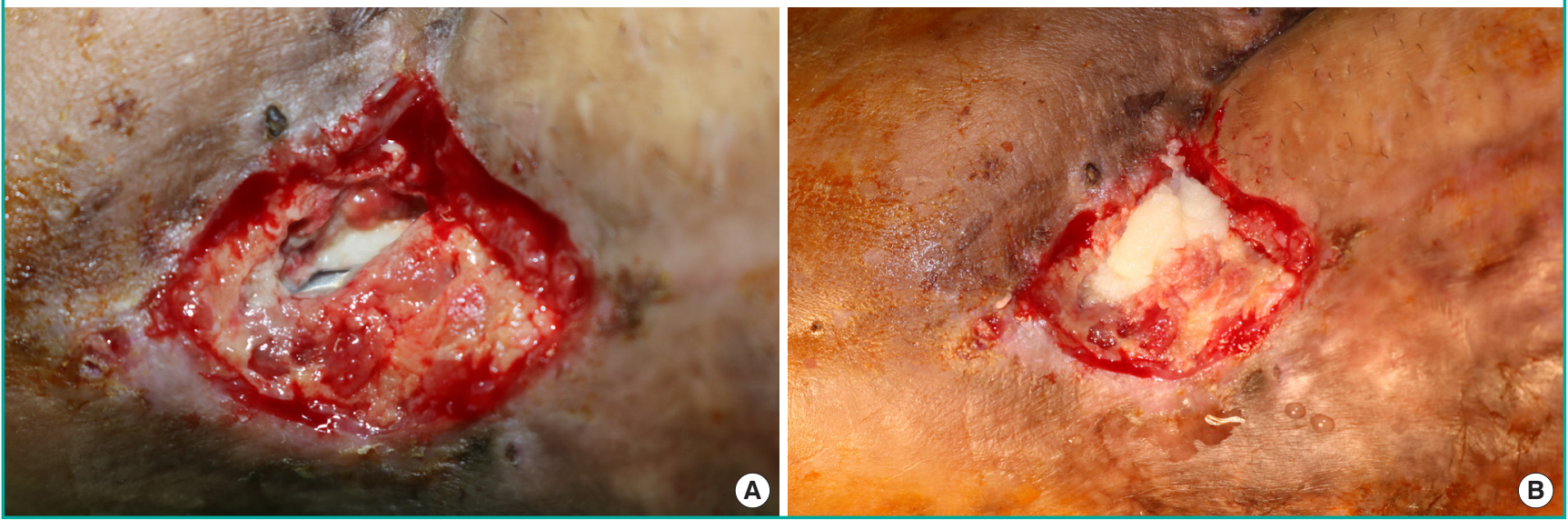

Table 1. Demographics of patients

\begin{tabular}{|c|c|c|c|c|c|c|c|c|c|c|c|}
\hline $\begin{array}{l}\text { Patient } \\
\text { no. }\end{array}$ & Sex & $\begin{array}{l}\text { Age } \\
\text { (yr) }\end{array}$ & Etiology & $\begin{array}{l}\text { Underlying } \\
\text { disease }\end{array}$ & $\begin{array}{l}\text { Site of } \\
\text { wound }\end{array}$ & $\begin{array}{c}\text { Wound } \\
\text { bed }\end{array}$ & $\begin{array}{c}\text { Goal of } \\
\text { application }\end{array}$ & $\begin{array}{l}\text { Dressing } \\
\text { method }\end{array}$ & $\begin{array}{c}\text { Wound } \\
\text { area } \\
\left(\mathrm{mm}^{2}\right)\end{array}$ & $\begin{array}{l}\text { Wound } \\
\text { depth } \\
\text { (mm) }\end{array}$ & $\begin{array}{l}\text { Amount } \\
\text { of CGPaste } \\
\text { applied } \\
(\mathrm{mL})\end{array}$ \\
\hline 1 & Male & 43 & Pressure ulcer & None & Trochanteric area & Granulation & Re-epithelialization & NPWT & $20 \times 15$ & 15 & 2 \\
\hline 2 & Male & 69 & Pressure ulcer & PAOD, DM & Lateral malleolus & Bone & Granulation growth & NPWT & $40 \times 30$ & 15 & 2 \\
\hline 3 & Female & 70 & Necrotizing fasciitis & DM, HTN & Lower leg & Granulation & Re-epithelialization & Wet & $20 \times 10$ & 5 & 2 \\
\hline 4 & Male & 63 & DFU & DM, ESRD & $\begin{array}{l}\text { 2nd web space of } \\
\text { the foot }\end{array}$ & Granulation & Re-epithelialization & Wet & $15 \times 5$ & 10 & 2 \\
\hline 5 & Female & 34 & Pressure ulcer & None & Coccyx & Bone & Granulation growth & Wet & $20 \times 20$ & 10 & 2 \\
\hline 6 & Male & 18 & Trauma (motorcycle) & None & Foot dorsum & Bone & Granulation growth & NPWT & $30 \times 20$ & 10 & 2 \\
\hline 7 & Female & 53 & Osteomyelitis & None & Ankle & Granulation & Granulation growth & Wet & $20 \times 20$ & 10 & 2 \\
\hline
\end{tabular}

of complications such as paste absorption or infection secondary to CGPaste application.

\section{RESULTS}

We analyzed seven patients who were treated with CGPaste. Our study included four males and three females (mean age, 50.0 years; range, $18-70$ years) with ulcerative wounds (Table $1)$. The causes of the chronic wounds were pressure ulcers (three patients, $42.86 \%$ ), diabetic foot ulcer (one patient, $14.29 \%$ ), necrotizing fasciitis (one patient, 14.29\%), mechanical trauma by traffic accident (one patient, 14.29\%), and osteomyelitis (one patient, 14.29\%). Four patients had no underlying medical disease, and the other three patients had PAOD and $\mathrm{DM}$, hypertension, or end-stage renal disease.

In three patients, CGPaste was applied to cover the exposed underlying bone and to promote the growth of granulation tissue infiltrate over the bony tissue. In the other three patients,
CGPaste was applied to ensure adequate coverage and re-epithelialization of ulcerative lesions, because well-granulated tissue was observed to have remained. CGPaste was applied to promote granulation tissue growth even though the wound bed contained granulation tissue in one patient. Due to osteomyelitis, this patient underwent bone resection of the talus and bone graft from the anterior superior iliac spine (ASIS). As the wound bed was unstable, the rate of granulation tissue growth was comparatively slow, and CGPaste was applied with the goal of promoting granulation tissue growth. After the application of paste-type ADM, application of NPWT was the primary method of dressing. However, only three of seven patients were treated with NPWT due to the cost, anatomical position of the wound, poor compliance of the patient, or an allergic reaction to the tape used for NPWT.

The mean wound area was $453.57 \mathrm{~mm}^{2}$ (range, $15 \times 5$ to $40 \times 30$ $\mathrm{mm}^{2}$ ), and the mean wound depth was $10.71 \mathrm{~mm}$ (range, 5-15 $\mathrm{mm}$ ). All patients in this study received $2 \mathrm{~mL}$ of CGPaste. 
Table 2. Results of application of CGPaste

\begin{tabular}{|c|c|c|c|c|c|c|c|}
\hline $\begin{array}{l}\text { Patient } \\
\text { no. }\end{array}$ & Sex & Age (yr) & $\begin{array}{l}\text { Achievement of } \\
\text { wound healing }\end{array}$ & $\begin{array}{l}\text { Duration of } \\
\text { healing (wk) }\end{array}$ & Complication & Infection & Seroma \\
\hline 1 & Male & 43 & Achieved & 4 & Paste resorption (partial) & - & - \\
\hline 2 & Male & 69 & Failed & NA & Paste resorption (complete) & - & - \\
\hline 3 & Female & 70 & Achieved & 2 & None & - & - \\
\hline 4 & Male & 63 & Achieved & 2 & None & - & - \\
\hline 5 & Female & 34 & Achieved & 2 & Paste resorption (partial) & - & - \\
\hline 6 & Male & 18 & Failed & NA & Paste resorption (complete) & - & - \\
\hline 7 & Female & 53 & Achieved & 2 & None & - & - \\
\hline
\end{tabular}

Among the seven patients studied, five patients (71.43\%) achieved the goal of applying CGPaste, while the treatment failed in the other two patients (28.57\%) (Table 2). The mean duration until complete healing in the five patients in whom treatment succeeded was 2.4 weeks. A patient with a pressure ulcer on his right trochanteric area, measuring $20 \times 15 \mathrm{~mm}^{2}$, with the wound bed showing granulation tissue, had the longest healing process (4 weeks). A patient with necrotizing fasciitis showed granulation tissue growth and re-epithelialization 2 weeks after paste application. A patient with a diabetic foot ulcer (wound area, $20 \times 20 \mathrm{~mm}^{2}$; wound bed, granulation tissue) in the second web space of his foot required 2 weeks to achieve complete wound healing. A patient with a coccygeal pressure ulcer (wound area, $20 \times 20 \mathrm{~mm}^{2}$; wound bed, bone) showed growth of granulation tissue 2 weeks after the application of paste-type ADM. A patient with osteomyelitis on the ankle (wound area, $20 \times 20 \mathrm{~mm}^{2}$; wound bed, granulation tissue) who received bone resection and autogenous bone graft from the ASIS required 2 weeks to achieve the goal of granulation tissue growth. One of the two patients who failed to achieve the goal was a patient with a pressure ulcer in the lateral malleolus, and the other had a soft tissue defect in the foot dorsum due to a traffic accident. The former was a patient with a pressure ulcer (wound area, $40 \times 30 \mathrm{~mm}^{2}$; wound bed, bone) with exposure of the fibula on the lateral malleolus, and CGPaste was used to promote the growth of granulation tissue. In the latter case, there was a defect on the patient's foot dorsum (wound area, $20 \times 20 \mathrm{~mm}^{2}$; wound bed, bone) with navicular bone exposure, and a paste-type $\mathrm{ADM}$ was used to promote the growth of granulation tissue.

During the period of wound healing, two patients showed minor resorption of the paste and two showed complete resorption. Among the three patients who had a bony wound bed, two showed complete resorption of the paste and the other showed partial resorption. The wounds observed in the patients who showed complete resorption had been caused by pressure ulcer and trauma. They measured $40 \times 30 \mathrm{~mm}^{2}$ and $30 \times 20 \mathrm{~mm}^{2}$ in area and were $15 \mathrm{~mm}$ and $10 \mathrm{~mm}$ deep, respectively, meaning that they could be categorized as large wounds in the context of the seven studied cases. No other complications such as infection and/or seroma were reported.

\section{Case presentation \\ Patient 1}

A 42-year-old man visited our clinic with a pressure ulcer on his right trochanteric area. A coverage operation was performed under general anesthesia using a deep femoral artery perforatorbased propeller flap to treat his grade IV pressure ulcer. An ulcerative wound defect was observed at the distal end of the flap because of excessive tension. At the proximal end of the flap, the ulcerative wound measured $20 \times 15 \mathrm{~mm}^{2}$ in area, and the wound bed was $15 \mathrm{~mm}$ deep (beneath the normal skin level) and showed granulation tissue. CGPaste was applied with the aim of replacing the ECM in the deep ulcerative defect. NPWT was applied to maintain the CGPaste at the site of application and to promote the growth of granulation tissue. NPWT was replaced every 3 to 4 days at bedside or in the operating room. The defect was covered with granulation tissue, and re-epithelialization was completed at 4 weeks after the application of CGPaste (Fig. 3).

\section{Patient 4}

A 63-year-old man visited the emergency department with pain and swelling on the dorsum of his left foot. He was diagnosed with a diabetic foot infection and abscess formation, and a ray amputation of his third toe was performed at the department of orthopedic surgery. He was subsequently transferred to the department of plastic and reconstructive surgery for coverage of the resulting soft tissue defect. A coverage operation was performed using a split-thickness skin graft (STSG), for which the de-vitalized tendons of the extensor digitorum longus of the second and fourth toes were removed. An STSG procedure was performed using Matriderm (a collagen-elastin matrix) for cov- 


\section{Fig. 3. CGPaste application on pressure ulcer}

A 43-year-old man with a wound measuring $20 \times 34 \mathrm{~mm}^{2}$ in area. The wound defect can be observed before injection (A), immediately after application (B), 2 weeks after application (C), and following re-epithelialization after 4 weeks (D).
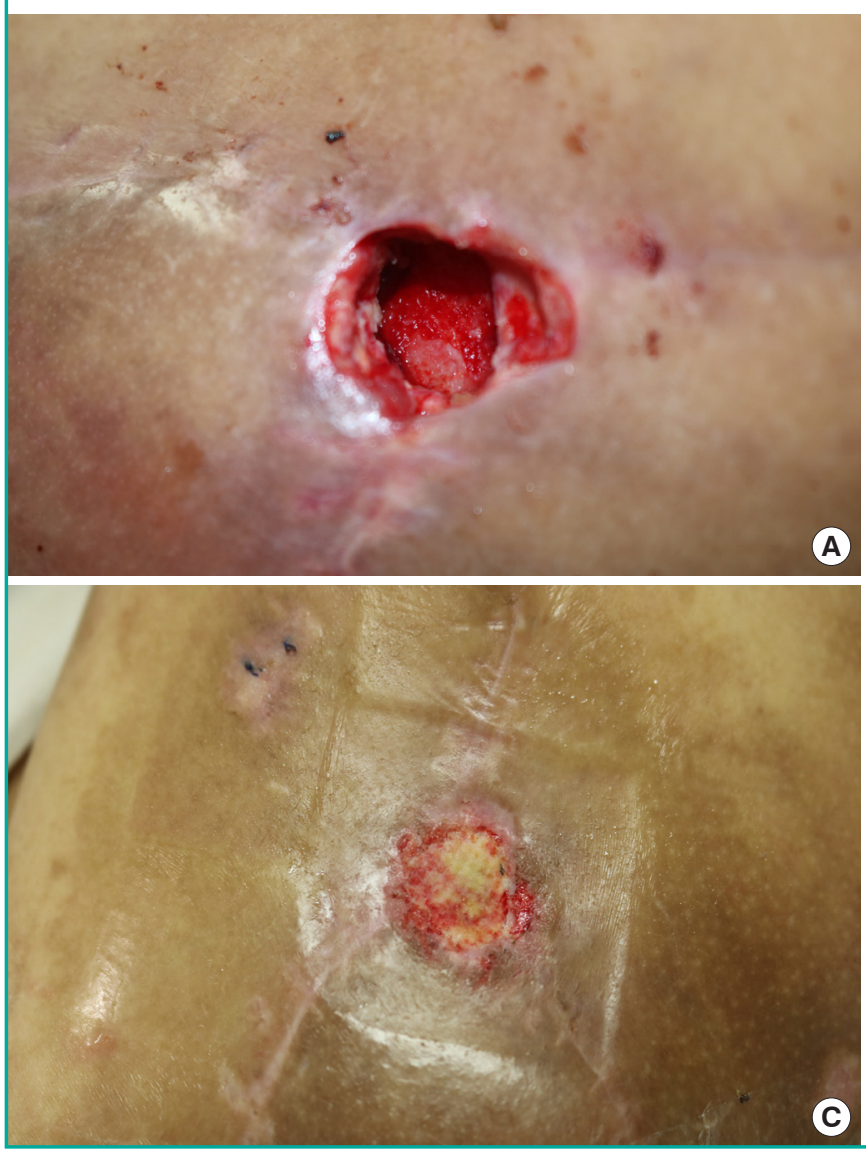

erage. The grafted skin was observed to have taken successfully, however, a small deep defect measuring $15 \times 5 \mathrm{~mm}^{2}$ in area was observed in the web space. CGPaste $(2 \mathrm{~mL})$ was applied to fill and cover this ulcerative wound. Further dressing changes were performed for 2 weeks, and the wound showed good coverage with healthy granulation tissue, following which the patient was discharged without any complications (Fig. 4).

\section{DISCUSSION}

Coverage of chronic ulcerative wounds is challenging, particularly in cases where the wounds are narrow and deep-seated with an insufficient blood supply. NPWT is useful for achieving coverage and promoting the growth of granulation tissue in such chronic ulcerative wounds [6]. Additionally, NPWT has been shown to effectively control surgical site infections [7]. However, NPWT usually requires a relatively long time to produce results, leading to prolonged hospitalization, which is a limitation of this therapy. Skin grafting can be a good option to achieve coverage of chronic deep wounds in cases where a local
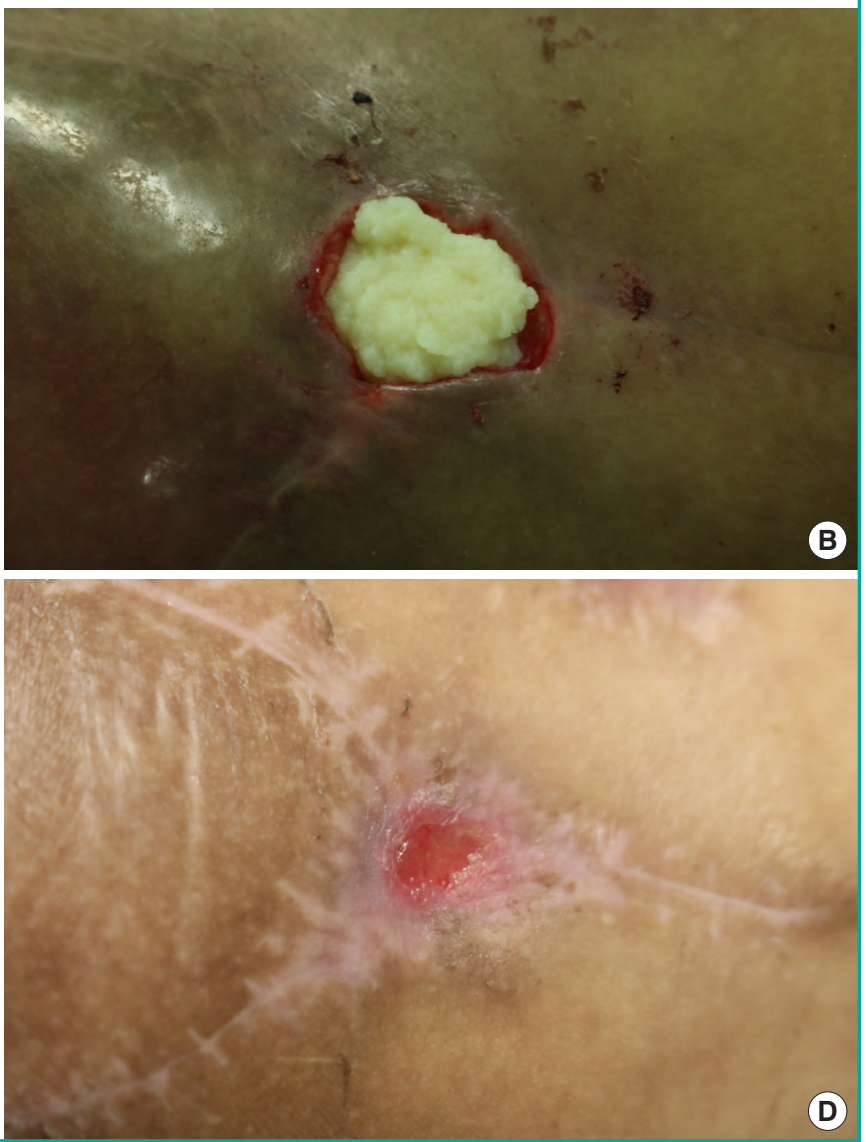

flap cannot be performed secondary to the lack of a viable perforator. However, appropriate engraftment requires adequate compression of the graft, which is challenging in narrow and deep wounds.

A few authors and investigators have advocated the use of collagen replacement materials, such as Matriderm (Dr. Otto Suwelack Skin \& Health Care AG, Billerbeck, Germany), which is extracted from bovine collagen and elastin, in small and deep chronic wounds. This product could be beneficial for application in deep dermal defects concomitantly with an STSG to improve the quality of the graft [8]. However, Matriderm cannot be used independently, and the application of Matriderm is not suitable for deep-seated wounds. Furthermore, Matriderm use is limited by the fact that it contains only collagenous components extracted from bovine tissue and does not offer the benefit of the usual support provided by healthy ECM.

Several authors have reported that beneficial effects were associated with the application of dermal substitutes for wound coverage. A single-center randomized controlled trial performed by Brigido [9] compared the application of human ADM to con- 


\section{Fig. 4. CGPaste application on diabetic foot ulcer}

A 63-year-old man with diabetic foot underwent ray amputation of his third toe. The wound can be observed on the dorsum of his foot, measuring $15 \times 5 \mathrm{~mm}^{2}$ in area, with a depth of $10 \mathrm{~mm}$. CGPaste was used on this wound. The wound defect can be observed before injection (A), immediately after application (B), 1 week after application (C), and following re-epithelialization after 2 weeks (D).
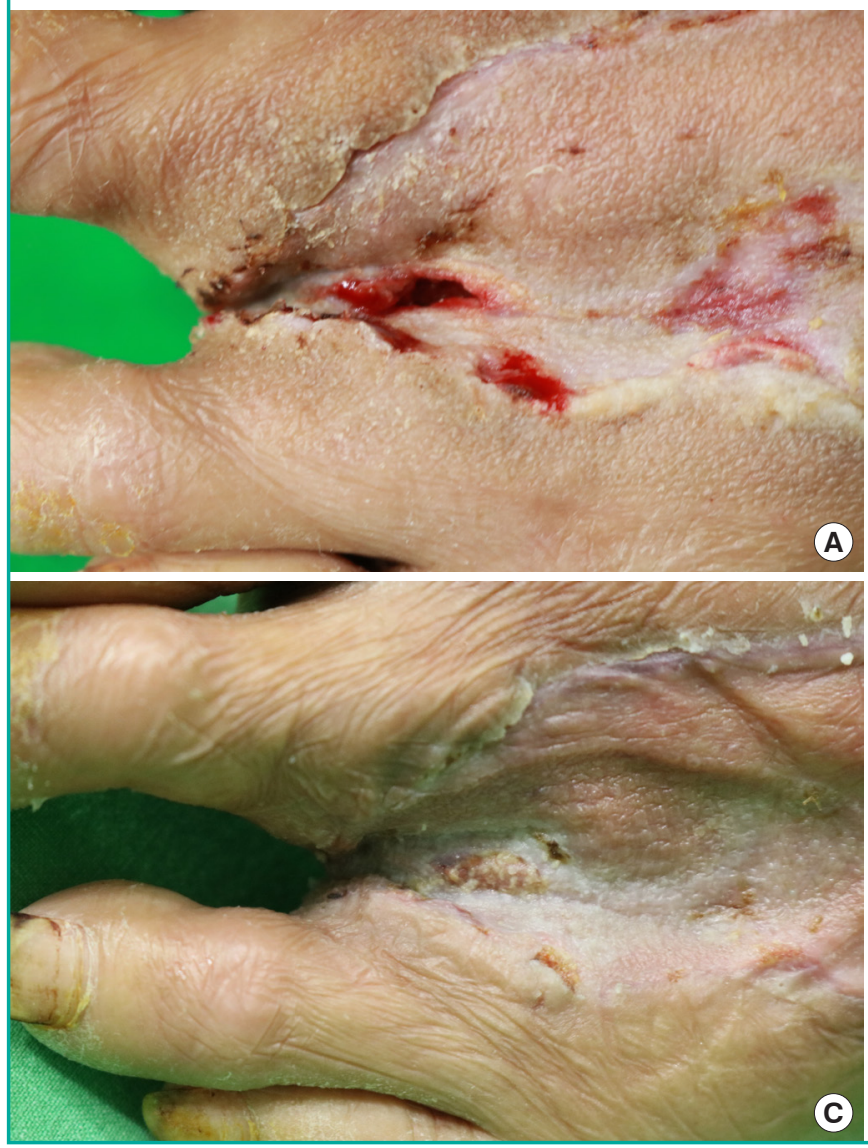

C

ventional methods using sharp debridement and gauze dressing in uninfected wounds of the lower extremity. They showed that 12 of 14 patients $(85.71 \%)$ in the ADM group achieved complete healing at the 16th week of treatment, whereas only four of 14 patients $(28.57 \%)$ in the control group achieved complete wound healing. Based on the previous literature, we hypothesized that the paste-type ADM would be helpful for the management of ulcerative wounds in instances where skin grafting or flap operations cannot be not easily performed.

In present study, there were two main goals of applying pastetype ADM. One was re-epithelialization of ulcerative wounds, leading to complete wound healing. The other goal was growth of granulation tissue above the avascular tissue, resulting in formation of the tissue basement, which would able to be covered with a skin graft later. We assumed that if the defect is not large, the paste-type $\mathrm{ADM}$ would provide an ECM scaffold and promote viable cell integration and vascularization from the surrounding host tissue. Therefore, interactions between the surrounding tissue and the paste-type $\mathrm{ADM}$ could potentially re-
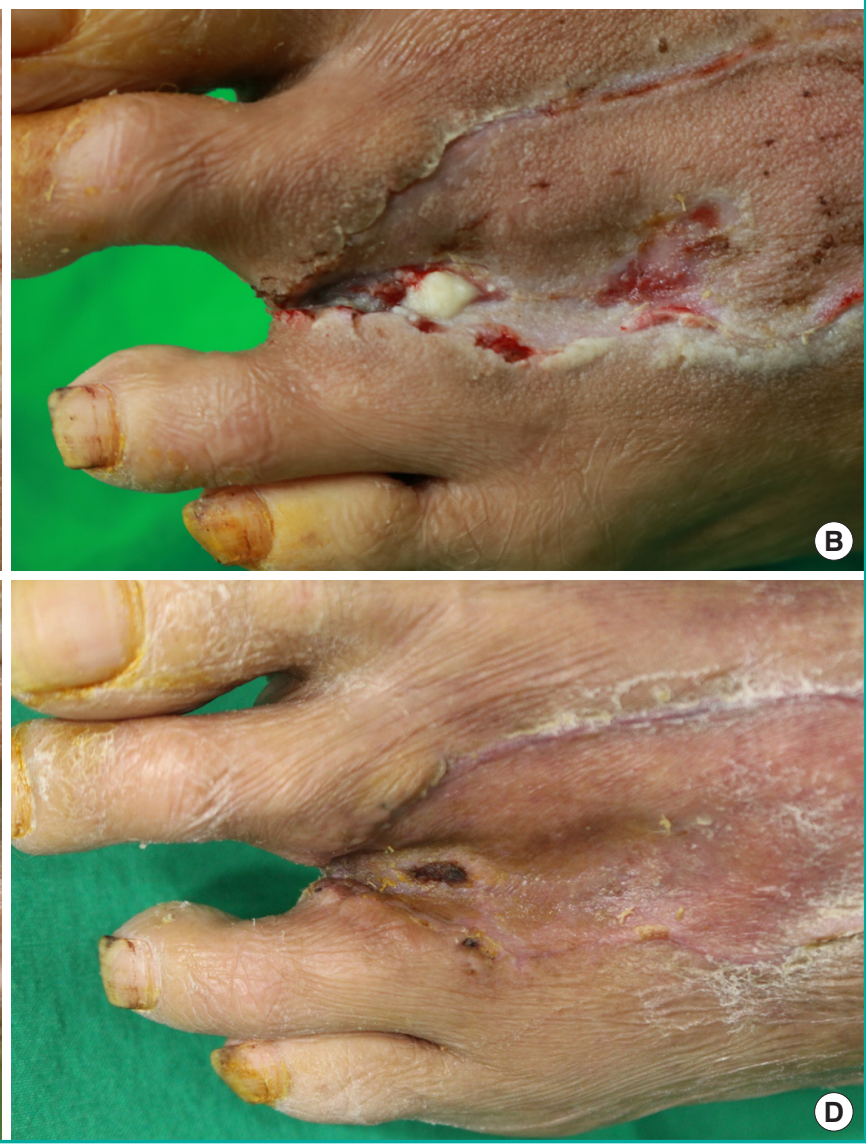

sult in wound healing by re-epithelialization or growth of granulation tissue above avascular bone or tendon. As a result, even in patients in whom the wound bed is bony or tendinous, if the wound is not very wide and is well debrided, CGPaste can show a "good take" to the wound bed, which makes it possible to provide a healthy wound bed to perform a subsequent coverage operation such as STSG. In this series, there was one patient with a small wound (about $20 \times 20 \mathrm{~mm}^{2}$ ) that showed granulation tissue growth above the bone tissue.

The appropriate application of paste-type ADM to a chronic wound is determined by multiple factors. First, wound size affects resorption of the paste. Among all patients analyzed, complete resorption was observed in two patients, who presented with relatively large and deep wounds (area, $40 \times 30 \mathrm{~mm}^{2}$ and $30 \times 20 \mathrm{~mm}^{2}$; depth, $15 \mathrm{~mm}$ and $10 \mathrm{~mm}$, respectively). It is challenging to achieve re-epithelialization or growth of granulation tissue in a wide defect only via application of paste-type ADM. Based on this experience, we emphasize that wound size and depth are important factors that ought to be considered in en- 
suring the engraftment of $\mathrm{ADM}$ paste, and that wounds measuring $<10 \times 10 \mathrm{~mm}^{2}$ and $<10 \mathrm{~mm}$ in depth are most likely to show good results. Second, the condition of the wound bed is an important factor. In this study, all four patients with a granulated wound bed showed complete engraftment of the ADM paste and achieved complete re-epithelialization or further growth of granulation tissue, whereas the three patients with a bony wound bed showed partial or complete resorption of the paste. Despite the wound bed, one of the three patients with a bony wound bed whose wound size was comparatively small $\left(20 \times 20 \mathrm{~mm}^{2}\right)$ showed good take of the ADM with granulation tissue growth, enabling a successful coverage operation later. CGPaste can be applied to deep and small chronic ulcerative wounds, which can help shorten the patient's length of hospitalization, promoting a faster recovery and return to normal life activities. Moreover, it could avoid the need for further operations performed under general anesthesia, which is particularly useful for avoiding complications in elderly patients.

The present study has several limitations. First, only a small number of patients was included in this study. However, this new material was introduced in 2017 and has not been widely commercialized yet. Therefore, we designed this case series with two major aims. The first was to demonstrate the specific clinical application of $\mathrm{ADM}$ paste to various kinds of wounds in terms of etiology, wound area/depth, and even the dressing method used. The second aim was to establish indications for the application of paste-type ADM in chronic wounds. Thus, although the sample size was small, we believe that our preliminary case series shows originality in that it presents specific cases of the application of paste-type $\mathrm{ADM}$ and indications for pastetype ADM. Second, because our study was retrospective, this paper was not able to assess outcomes objectively, using a standardized analysis of patients and wound type. Moreover, there is no precise definition of a "chronic wound," although this term usually refers to a wound that does not respond to treatment for more than 3 months. Therefore, various causes can lead to chronic wounds, and it is difficult to standardize this aspect without knowing for which wound type the paste-type ADM works better. Therefore, we tried to establish standardized indications for paste-type ADM after applying it to various wounds, rather than standardizing the process before application. Further long-term and large-scale studies are certainly warranted to establish the effectiveness of CGPaste. The final major limitation is related to the difference in the effects of CGPaste according to the dressing method. We used paste-type ADM followed by NPWT as the first-line application, but if not possible, we applied a wet gauze dressing. Therefore, we acknowledge that there may have been differences in the effect of paste-type $\mathrm{ADM}$ according to which dressing method was applied. However, the chronic wounds analyzed in this study were largely refractory to NPWT and conventional dressing methods, leading us to suggest that the choice of the dressing method after applying ADM is unlikely to have been an especially crucial factor. We also think that it is necessary to conduct larger-scale research in the future to further clarify the effect of the choice of dressing material after application of paste-type $\mathrm{ADM}$.

Nevertheless, to our knowledge, the present study is the first to present preliminary results following the application of $\mathrm{ADM}$ paste to chronic wounds.

We conclude that a paste-type ADM, CGPaste, was effective in treating small, deep, chronic ulcerative wounds measuring $<10 \times 10 \mathrm{~mm}^{2}$ with granulation tissue-based wound beds. Paste-type ADM is a useful option for wound coverage in patients in whom skin grafting or flap operations are challenging.

\section{NOTES}

\section{Conflict of interest}

No potential conflict of interest relevant to this article was reported.

\section{Ethical approval}

The study was approved by the Institutional Review Board of Sanggye Paik Hospital (approval number: 2018-08-028) and performed in accordance with the principles of the Declaration of Helsinki. Written informed consents were obtained.

\section{Patient consent}

The patients provided written informed consent for the publication and the use of their images.

\section{REFERENCES}

1 Braddock M. Tissue repair and ulcer/wound healing-Institut Pasteur Euroconference: molecular mechanisms, therapeutic targets and future directions. IDrugs 2005;8:381-3.

2. Kirsner RS, Bohn G, Driver VR, et al. Human acellular dermal wound matrix: evidence and experience. Int Wound J 2015; 12:646-54.

3. Cazzell S, Vayser D, Pham H, et al. A randomized clinical trial of a human acellular dermal matrix demonstrated superior healing rates for chronic diabetic foot ulcers over conventional care and an active acellular dermal matrix comparator. Wound Repair Regen 2017;25:483-97.

4. Martin BR, Sangalang M, Wu S, et al. Outcomes of allogenic acellular matrix therapy in treatment of diabetic foot wounds: 
an initial experience. Int Wound J 2005;2:161-5.

5. Jang HJ, Kim YM, Yoo BY, et al. Wound-healing effects of human dermal components with gelatin dressing. J Biomater Appl 2018;32:716-24.

6. Green T, Kavros S, Springer S, et al. Team approach: complex dermal wound-healing utilizing negative-pressure wound therapy (NPWT) in orthopaedic trauma. JBJS Rev 2018;6:e1.

7. Jeffery S, Leaper D, Armstrong D, et al. Using negative pres- sure wound therapy to prevent surgical site infection. J Wound Care 2018;27(Suppl 3):S5-13.

8. Choi JY, Kim SH, Oh GJ, et al. Management of defects on lower extremities with the use of matriderm and skin graft. Arch Plast Surg 2014;41:337-43.

9. Brigido SA. The use of an acellular dermal regenerative tissue matrix in the treatment of lower extremity wounds: a prospective 16-week pilot study. Int Wound J 2006;3:181-7. 